\title{
Generation of platelet-derived microparticles in patients undergoing cardiac surgery is not affected by complement activation
}

\author{
Jeanette $\mathrm{M}$. van den Goor, $\mathrm{MSc}, \mathrm{CP}^{\mathrm{a}}$ \\ Albert van den Brink, MD \\ Rienk Nieuwland, $\mathrm{PhD}^{\mathrm{c}}$ \\ Willem van Oeveren, $\mathrm{PhD}^{\mathrm{e}}$ \\ Peter M. Rutten, $C P^{a}$ \\ Robert Tepaske, MD \\ Jan G. Tijssen, $\mathrm{PhD}^{\mathrm{d}}$ \\ Augueste Sturk, $\mathrm{PhD}^{\mathrm{c}}$ \\ Bas A. de Mol, MD, PhD \\ León Eijsman, $\mathrm{MD}, \mathrm{PhD}^{\mathrm{a}}$
}

\footnotetext{
From the Departments of Cardio-thoracic Surgery, ${ }^{\mathrm{a}}$ Intensive Care, ${ }^{\mathrm{b}}$ Clinical Chemistry, ${ }^{\mathrm{c}}$ and Cardiology, ${ }^{\mathrm{d}}$ Academic Medical Center of the University of Amsterdam, Amsterdam, The Netherlands, and HaemoProbe, ${ }^{\mathrm{e}}$ Groningen, The Netherlands.

Supported by Medtronic, Minneapolis, Minn.

Received for publication Dec 20, 2002; revisions requested Jan 21, 2003; revisions received Feb 17, 2003; accepted for publication June 16, 2003.

Address for reprints: Jeanette M. van den Goor, MSc, CP, Department of Cardio-thoracic Surgery, Academic Medical Center of the University of Amsterdam, Meibergdreef 9, 1105 AZ Amsterdam, The Netherlands (E-mail: J.M.vandenGoor@AMC. UVA.nl).

J Thorac Cardiovasc Surg 2003;126:1101-6

Copyright () 2003 by The American Association for Thoracic Surgery

$0022-5223 / 2003 \$ 30.00+0$

doi:10.1016/S0022-5223(03)01031-6
}

Objective: The mechanisms causing the presence of platelet-derived microparticles in the circulation are unknown. In vitro platelets release platelet-derived microparticles in response to complement activation. This study evaluates the relationship between complement activation and levels of circulating platelet-derived microparticles in patients undergoing cardiac surgery.

Methods: Prospectively, 71 patients were included who underwent elective coronary artery bypass grafting with cardiopulmonary bypass. The patients were randomly allocated to one of the 3 groups: uncoated oxygenator, UnModified Surface $(n=25)$ or oxygenator coated with either BioPassive Surface $(n=25)$ or BioActive Surface $(\mathrm{n}=21)$. Platelet-derived microparticles and terminal complement complexes were determined before bypass and after induction of anesthesia, 15 minutes after the start of cardiopulmonary bypass, at the end of cardiopulmonary bypass, and 30 minutes after administration of protamine sulfate.

Results: Demographic and cardiopulmonary bypass data were similar for the 3 groups. At the end of cardiopulmonary bypass, platelet-derived microparticle numbers were decreased in all 3 groups. No significant differences were observed among the groups at any sampling point. At the end of cardiopulmonary bypass, terminal complement complex concentrations were increased in all groups $(P<.001)$, and significant differences among the groups were present $(P=.002)$.

Conclusions: Despite significant complement activation, no increase in numbers of circulating platelet-derived microparticles was found in the systemic blood of patients undergoing cardiac surgery with cardiopulmonary bypass. Thus complement activation in vivo does not necessarily affect generation of platelet-derived microparticles.

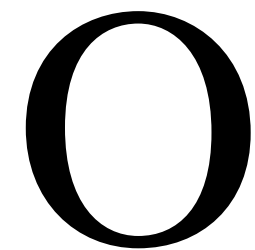

$\mathrm{n}$ activation and during apoptosis, platelets and other cells bud off small parts of their plasma membrane, the so-called microparticles. Extensive in vitro studies have been reported on plateletderived microparticles (PMPs). ${ }^{1,2}$ When platelets are stimulated in vitro with agonists, such as a combination of $\alpha$-thrombin and collagen, the complement complex C5b-9, or the $\mathrm{Ca}^{2+}$ ionophore A23187, they release large numbers of PMPs. ${ }^{1,3,4}$ PMPs possess platelet factor 3 activity; that is, they facilitate coagulation through exposure of negatively charged phospholipids, thereby providing binding sites for activated coagulation factors $\mathrm{V}$ (factor Va), VIIIa, IXa, and XIa ${ }^{1,2,5,6}$ and enabling the formation of tenase and prothrombinase complexes. ${ }^{1,2,7}$ 
TABLE 1. Demographic and CPB data

\begin{tabular}{lccc}
\hline & \multicolumn{3}{c}{ Surface modification of the oxygenator } \\
\cline { 2 - 4 } & UMS & BPS & BAS \\
\hline Patient no. & 25 & 25 & 21 \\
Male/female & $25 / 0$ & $23 / 2$ & $20 / 1$ \\
Age (y) & $62.0 \pm 7.1^{1}$ & $59.9 \pm 9.7$ & $61.4 \pm 9.9$ \\
Weight (kg) & $87.5 \pm 12.1$ & $87.1 \pm 14.8$ & $83.1 \pm 9.8$ \\
Body surface area $\left(\mathrm{m}^{2}\right)$ & $2.04 \pm 0.17$ & $2.06 \pm 0.18$ & $1.99 \pm 0.12$ \\
Hemoglobin (mmol/L) & $8.5 \pm 0.6$ & $8.4 \pm 0.7$ & $8.3 \pm 0.5$ \\
Platelet count (10 $/ \mathrm{L})$ & $227 \pm 67.4$ & $219 \pm 69$ & $233 \pm 53$ \\
Cardiopulmonary & $93.5 \pm 21.8$ & $85.1 \pm 21.4$ & $95.4 \pm 24.2$ \\
$\quad$ bypass (min) & & & \\
Aortic crossclamping & $58.5 \pm 15.9$ & $55.1 \pm 17.2$ & $58.6 \pm 19.7$ \\
$\quad$ time (min) & & & \\
\hline
\end{tabular}

All data are presented as means $\pm \mathrm{SD}$. No significant differences were found among the groups for any of the variables.

Increased numbers of PMPs have been reported in the circulation of patients with diabetes, ${ }^{8,9}$ patients undergoing cardiopulmonary bypass (CPB) ${ }^{10,11}$ and patients with acute coronary ischemia, ${ }^{12}$ heparin-induced thrombocytopenia, ${ }^{13}$ myocardial infarction, ${ }^{14}$ uremia, ${ }^{15}$ idiopathic thrombocytopenic purpura, ${ }^{16}$ disseminated intravascular coagulation, ${ }^{17}$ plasmapheresis, ${ }^{18}$ or meningococcal septic shock, ${ }^{19}$ which are all diseases that have been associated with a thromboembolic tendency. Increased numbers of PMPs have been found also in the pericardial fluid of patients undergoing surgical intervention with $\mathrm{CPB} .^{20}$

Despite the widespread presence of PMPs in the circulation, however, the mechanisms causing their release in vivo are still unknown. Complement activation is markedly increased during $\mathrm{CPB}$, whereas coatings are known to reduce complement activation. Generation of PMPs induced by complement activation was anticipated. Therefore in the present study the relationship between in vivo complement activation and the concentrations of circulating PMPs was evaluated in patients undergoing cardiac surgery with CPB with noncoated oxygenators and coated oxygenators.

\section{Materials and Methods \\ Patients}

On approval of the Medical Ethics Committee of the Academic Medical Center and obtainment of signed informed consent, we prospectively included 71 patients who underwent elective coronary artery bypass grafting with CBP. Inclusion criteria were age 21 to 75 years, elective coronary artery bypass surgery, ejection fraction of greater than $30 \%$, body surface area of greater than 1.66 $\mathrm{m}^{2}$, and a preoperative hemoglobin value of greater than 7.5 $\mathrm{mmol} / \mathrm{L}$. Exclusion criteria were combined valve surgery or aneurysmectomy, redo operations, insulin-dependent diabetes mellitus, creatinine plasma level of greater than $300 \mu \mathrm{mol} / \mathrm{L}$, preoperative intra-aortic balloon pumping, preoperative use of nonsteroidal anti-inflammatory drugs, preoperative use of warfarin, preopera- tive immunosuppressive therapy of greater than 24 hours' duration, allergic reactions, and chronic obstructive pulmonary disease. The patients were randomly allocated to one of 3 groups (Table 1).

\section{CPB}

All extracorporeal bypass circuits consisted of a hollow-fiber oxygenator (Affinity; Medtronic, Minneapolis, Minn). The oxygenator was uncoated (ie, without surface modification, UnModified Surface [UMS; $\mathrm{n}=25]$ ) or coated with either BioPassive Surface (BPS; $\mathrm{n}=25$; Trillium, Medtronic) or BioActive Surface (BAS; $\mathrm{n}$ = 21; Carmeda, Medtronic). The additional noncoated components of the extracorporeal circuit were identical for all patients and included a soft-shell venous reservoir, 2 additional reservoirs, an arterial line filter, a tubing system (Medtronic), and a roller pump as an arterial blood pump (3M Sarns, Ann Arbor, Mich). One of the additional reservoirs was used to collect the shed blood, which was processed by a cell saver before being returned into the systemic circulation after termination of the bypass procedure. The other reservoir was used to collect left vent (systemic) blood, which was returned into the systemic circulation through the soft-shell venous reservoir during the bypass procedure. The extracorporeal system was primed with $500 \mathrm{~mL}$ of Ringer lactate solution, $1 \mathrm{~L}$ of Haemaccell (Behring, Malburg, Germany), 100 $\mathrm{mL}$ of mannitol $20 \%$ (wt/vol), $50 \mathrm{~mL}$ of sodium bicarbonate $8.4 \%$ (wt/vol), and $200 \mathrm{~mL}$ of aprotinin $\left(2 \times 10^{6} \mathrm{KIU}\right.$ Trasylol; Bayer, Leverkusen, Germany). Magnesium sulfate, $4 \mathrm{mmol} / 10 \mathrm{~kg}$ (ie, 24 $\geq x \leq 32 \mathrm{mmol}$ ) and 10,000 IU of bovine heparin (Leo Pharmaceutical Products, Weesp, The Netherlands) were added to the priming solution. The total priming volume was $1.85 \mathrm{~L}$. All patients received $300 \mathrm{IU} / \mathrm{kg}$ heparin (Leo Pharmaceutical Products) before cannulation of the aorta. CPB was initiated when the activated clotting time was 480 seconds or greater. During CPB, the activated clotting time was maintained at greater than 480 seconds by means of administration of additional heparin when required. Moderate hypothermia $\left(30^{\circ} \mathrm{C}-34^{\circ} \mathrm{C}\right)$ was used for all patients. Myocardial protection was achieved by using cold $\left(4^{\circ} \mathrm{C}-\right.$ $8^{\circ} \mathrm{C}$ ) crystalloid cardioplegia solution (St Thomas). Shed blood in the surgical field was processed with a cell saver (HaemoLite 2 plus; Haemonetics Corp, Braintree, Mass). Processed blood was returned into the systemic circulation of the patients immediately after CPB. After weaning from $\mathrm{CPB}$ and decannulation, heparin was neutralized with protamine sulfate at a 1:1 ratio.

\section{Collection of Blood Samples}

Arterial blood samples were obtained before induction of anesthesia, 15 minutes after the start of $\mathrm{CPB}$, at the termination of $\mathrm{CPB}$, and 30 minutes after protamine administration. For comparison between plasma samples, PMP numbers, platelet counts, and terminal complement complex (TCC) concentrations were corrected for hemodilution by hemoglobin concentration.

\section{Cell Count}

Blood samples for hemoglobin and platelet counts were collected in 5-mL glass vacutainer tubes containing ethylenediamine tetraacetic acid (Becton Dickinson, San Jose, Calif) and analyzed on a Celldyn 4000 (Abbot, Mijdrecht, The Netherlands). 


\section{TCCs}

Arterial blood (2 mL) was anticoagulated with $10 \mathrm{mmol} / \mathrm{L}$ ethylenediamine tetra-acetic acid. Cell-free plasma aliquots $(1 \mathrm{~mL})$ were prepared by means of centrifugation $(11$ minutes at $1100 \mathrm{~g}$ and $4{ }^{\circ} \mathrm{C}$ ) and stored at $-80^{\circ} \mathrm{C}$ until use. TCC measures the TCC bound to the S-protein, representing the soluble nonlytic form of TCC. TCC was determined by means of enzyme-linked immunoassay (Quidel, San Diego, Calif).

\section{PMPs}

Arterial blood $(4.5 \mathrm{~mL})$ was collected into $3.2 \%$ trisodium citrate (Becton Dickinson). Blood cells were removed by means of centrifugation for 20 minutes at $1550 \mathrm{~g}$ and room temperature, and plasma aliquots $(250 \mu \mathrm{L})$ were snap-frozen in liquid nitrogen and stored at $-80^{\circ} \mathrm{C}$ until use. After thawing plasma aliquots on melting ice, plasma was centrifuged for 30 minutes $(17,570 \mathrm{~g}$ and $20^{\circ} \mathrm{C}$ ) to pellet the microparticles, as described previously. ${ }^{19-22}$ After removal of $225 \mu \mathrm{L}$ of (MP-free) plasma, the $25-\mu \mathrm{mL}$ microparticle-enriched plasma was diluted with $225 \mu \mathrm{mL}$ of phosphate-buffered saline (PBS; $154 \mathrm{mmol} / \mathrm{L} \mathrm{NaCl}$ and $1.4 \mathrm{mmol} / \mathrm{L}$ phosphate, $\mathrm{pH}$ 7.4) containing $10.9 \mathrm{mmol} / \mathrm{L}$ trisodium citrate. Microparticles were resuspended and centrifuged (30 minutes at $17,570 \mathrm{~g}$ and $20^{\circ} \mathrm{C}$ ). Again, $225 \mu \mathrm{L}$ of the supernatant was removed, and microparticles were resuspended in the remaining 25 $\mu \mathrm{L}$ and diluted 4-fold with PBS/citrate buffer, of which $5 \mu \mathrm{L}$ was used per flow cytometric determination.

\section{Flow Cytometric Analysis}

Microparticle samples were analyzed in a FACSCalibur flow cytometer with CellQuest software (Becton Dickinson). PMPs were identified on forward scatter, side scatter, and binding of both phycoerythrin-labeled annexin V (PharMingen, San Jose, Calif) and fluorescein isothiocyanate-labeled anti-CD61 (glycoprotein IIIA; clone Y2/51, IgG1; Dako A/S, Glostrup, Denmark) to distinguish PMPs from events caused by noise. To identify annexin $\mathrm{V}$-positive microparticles, a fluorescence threshold was placed in a microparticle sample prepared without addition of calcium to correct for autofluorescence. To identify CD61-positive events, microparticles were incubated with a similar concentration of isotype-matched control antibody (fluorescein isothiocyanate-labeled IgG1; Becton Dickinson) to set the fluorescence threshold. Microparticles $(5 \mu \mathrm{L})$ were diluted in $35 \mu \mathrm{L}$ of PBS containing 2.5 $\mathrm{mmol} / \mathrm{L} \mathrm{CaCl}_{2}(\mathrm{pH} \mathrm{7.4)}$ and $5 \mu \mathrm{L}$ of 500 -fold prediluted normal mouse serum (Central Laboratory of the Netherlands Red Cross Bloodtransfusion Service, Amsterdam, The Netherlands). After incubation for 15 minutes at room temperature, annexin $\mathrm{V}(5 \mu \mathrm{L})$ plus anti-CD61 $(5 \mu \mathrm{L})$ or IgG1 control antibody was added. The mixtures were incubated in the dark (15 minutes at room temperature). Subsequently, $200 \mu \mathrm{L}$ of PBS/calcium buffer was added, and the suspensions were centrifuged (30 minutes at $17,570 \mathrm{~g}$ and $20^{\circ} \mathrm{C}$ ). Finally, $200 \mu \mathrm{L}$ of (MP-free) suspension was removed. The microparticles were diluted in $300 \mu \mathrm{L}$ of PBS/calcium buffer before flow cytometry, and all samples were analyzed for 1 minute. To estimate the number of PMPs per liter of plasma, the number of events $(\mathrm{N})$ found in the upper right (marker and annexin $\mathrm{V}$ positive) quadrant of the flow cytometric analysis (FL1 vs FL2) was used in the following formula: Number $/ L=N \times[100 / 5] \times$ $[355 / 150] \times\left[10^{6} / 250\right]$.

\section{Statistics}

Data were analyzed by using SPSS, release 11.0 (SPSS, Chicago, Ill). Demographic and CPB data are reported as means with SDs. Outcome data (PMPs, platelets, and TCCs) were corrected for hemodilution (hemoglobin) and are presented as medians with interquartile ranges. For all outcome variables, statistical analyses were performed on the change of that variable relative to the baseline value $(\mathrm{t}=0)$ per patient. Statistical significance $(P<.05$, 2 -sided) is indicated. The sample size was chosen on the basis of preliminary observations, indicating that approximately 25 patients per group would be sufficient to achieve statistically significant differences in blood activation between coated and noncoated oxygenators. Comparisons over time within treatment groups were made by applying the Wilcoxon signed-rank test to the (paired) observations at baseline and at the end of CPB. Comparisons between (treatment) groups were made by applying the KruskalWallis test to the change between the end of CPB and baseline. The observations at 15 minutes after the start of CPB and at 30 minutes after protamine sulfate are presented for descriptive purposes.

\section{Results}

\section{Clinical Results}

The coating groups (UMS, BPS, and BAS) were compared for preoperative parameters, including body surface area, age, sex, hemoglobin value, platelet count, and surgical data (CPB time and aortic crossclamping time). No significant differences were found among the groups for any of the variables tested (Table 1). There were no differences among the groups in hemoglobin concentrations and platelet counts at any sampling time. Hemodilution was similar in the 3 coating groups: the decreases in hemoglobin concentrations and platelet counts after the start of the CPB procedure were most likely due to hemodilution of the systemic patient blood by the priming volume of the extracorporeal circuit and administration of the cardioplegia solution for myocardial protection. In addition, in all 3 coating groups, a similar decrease in platelet count was observed after administration of protamine sulfate.

\section{PMPs and Platelet Counts During CPB}

The numbers of PMPs at baseline were similar among the 3 coating groups (Figure 1, A). Fifteen minutes after the start of $\mathrm{CPB}$, the PMP numbers decreased in all 3 groups, despite correction for hemodilution. During CPB, the PMP numbers slightly recovered, but at the end of bypass, they were still less than before the start of CPB. At the end of bypass, there was a significant decrease of PMPs in the UMS group $(P<$ $.001)$ and the BPS group $(P=.028)$ when compared with baseline values. After administration of protamine sulfate, PMP numbers again slightly decreased in all groups. Significant differences were not observed among the groups at any sampling point.

In contrast to PMP numbers, platelet counts significantly increased during $\mathrm{CPB}$ when corrected for hemodilution 


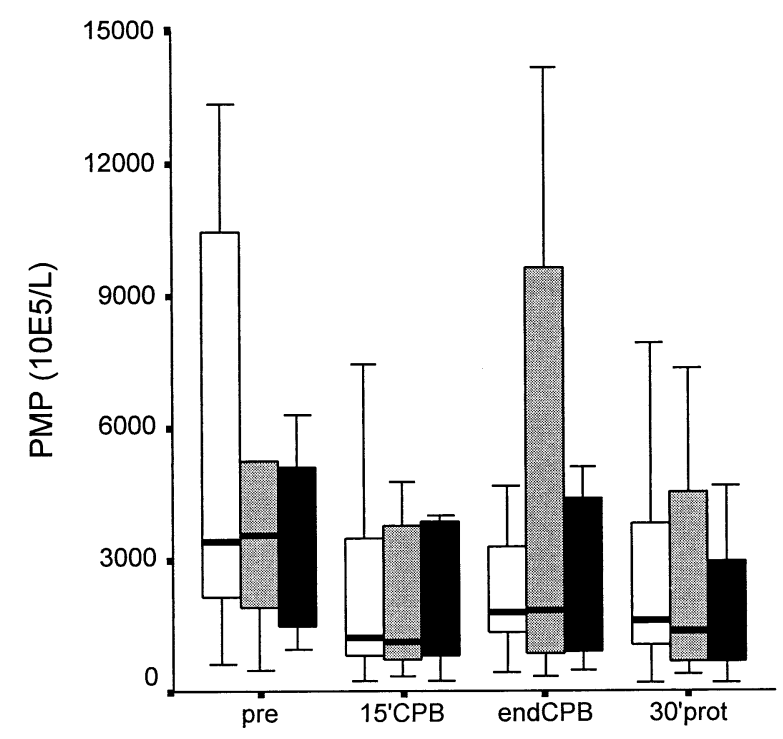

A

Sample time

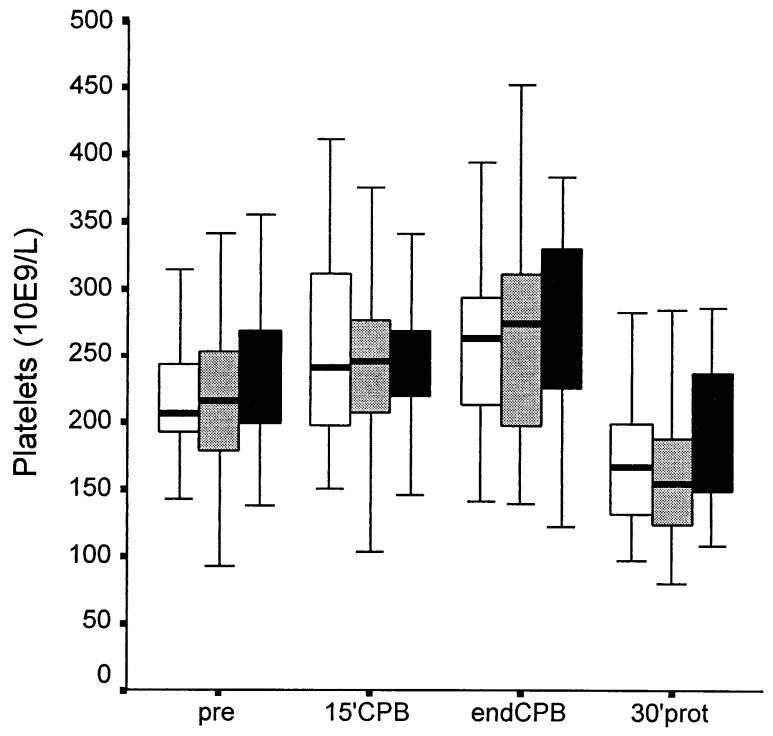

B

Sample time

Figure 1. Systemic concentrations of PMPs and platelet counts in patients undergoing CPB. Concentrations of PMPs and platelet counts before bypass and after induction of anesthesia (baseline value; pre), 15 minutes after the start of bypass $\left(15^{\prime}(P B)\right.$, at the end of bypass and after release of the aortic crossclamp (end $C P B$ ), and 30 minutes after administration of protamine sulfate (30'prot) are shown. The measurements were made in 25 patients treated with an uncoated oxygenator (ie, without Surface Modification, UMS group, open bars), 25 patients treated with an oxygenator coated with a BioPassive Surface (BPS group, shaded bars), and 21 patients treated with an oxygenator coated with a BioActive Surface (BAS group, striped bars). PMP concentrations $(A)$ and platelet counts $(B)$ were determined as described in the "Methods" section. No significant differences were present among the 3 oxygenator groups. Medians and interquartile ranges are presented.

(Figure 1, $B$; end of CPB: UMS group, $P=0.004$; BPS group, $P<.001$, and BAS group, $P=.003$ ).

\section{Complement Activation During CPB}

Before CPB, complement activation was not detectable or hardly detectable (Figure 2). Fifteen minutes after the start of CPB, plasma concentrations of TCCs increased in all 3 coating groups, indicating complement activation. TCC concentrations further increased during $\mathrm{CPB}$ and remained high after protamine sulfate administration. At the end of $\mathrm{CPB}, \mathrm{TCC}$ concentrations were significantly increased in all 3 groups $(P<.001)$ when compared with baseline values. At the end of CPB, significant differences among the coating groups were present $(P=.002)$. At the other sample times, no significant differences among the coating groups were found.

\section{Discussion}

This study shows that despite considerable activation of the complement system in vivo, circulating PMP numbers did not increase. These results were not anticipated because earlier in vitro studies clearly showed that complement activation triggers the release of PMPs. ${ }^{1,4,23-25}$ Our present data, however, suggest that other mechanisms must be involved in the release of microparticles in vivo. On the other hand, we cannot exclude that complement activation might contribute to microparticle release in other patient populations or that it might affect the release of microparticles in concert with other stimuli.

Earlier studies reported that CPB triggers the formation of PMPs both in vitro ${ }^{26}$ and in vivo. ${ }^{10,11}$ In contrast, we found an intraoperative decrease in PMP numbers. Most likely, this lack of increase is due to the many relatively recent improvements of the extracorporeal circuits. ${ }^{27-29} \mathrm{Al}-$ ternatively, in the present study the pericardial blood was not reinfused during the bypass procedure but rather afterward. Because pericardial blood contains relatively high numbers of cell-derived microparticles itself and is highly activated with regard to fibrinolysis and coagulation, one could hypothesize that by not returning this blood into the systemic circulation during bypass, one of the triggers for (systemic) blood activation has been eliminated. The same 


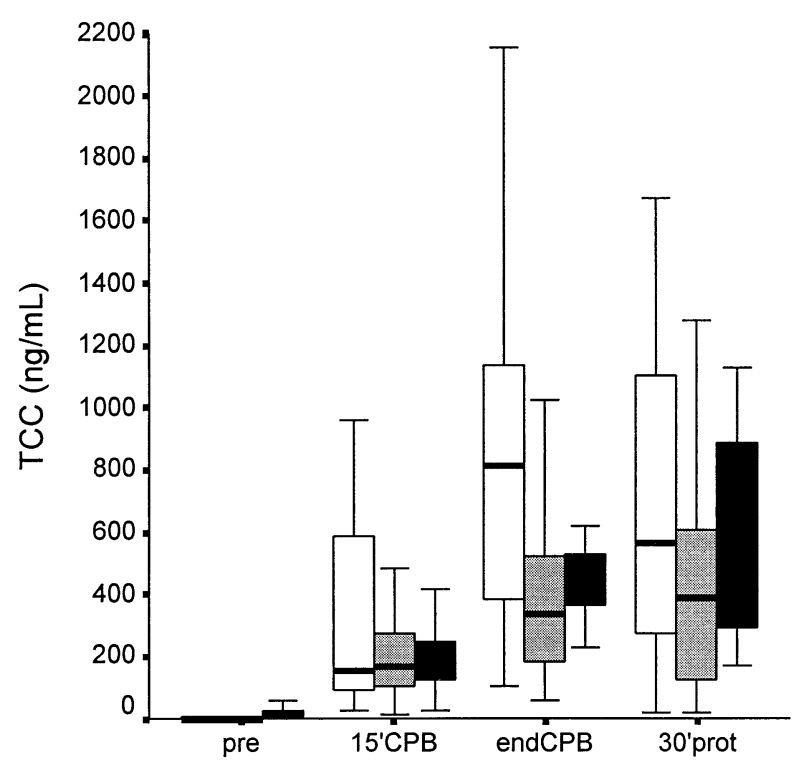

\section{Sample time}

Figure 2. Concentrations of systemic TCCs in patients undergoing CPB. TCC concentrations before bypass and after induction of anesthesia (baseline value, pre), 15 minutes after the start of bypass (15'CPB), at the end of bypass and after release of the aortic crossclamp (end $C P B$ ), and $\mathbf{3 0}$ minutes after administration of protamine sulfate (30'prot) are shown. The measurements were made in 25 patients treated with an uncoated oxygenator (ie, without Surface Modification (UMS group, open bars), 25 patients treated with an oxygenator coated with a BioPassive Surface (BPS group, shaded bars), and 21 patients treated with an oxygenator coated with a BioActive Surface (BAS group, striped bars) and performed as described in the "Methods" section. Medians and interquartile ranges are presented.

holds for platelet activation. Whereas earlier studies reported extensive platelet activation during CPB, more recent studies showed less platelet activation. ${ }^{27,30}$ For instance, Kestin and colleagues ${ }^{30}$ reported that platelets from the systemic circulation of patients undergoing CPB showed normal reactivity in vitro, had no loss of surface glycoprotein complexes Ib-IX and IIb-IIIa, and had hardly degranulated.

In contrast, pericardial blood contains relatively high numbers of PMPs and erythrocyte-derived microparticles. $^{20,27}$ Whether local complement activation (ie, in this wound blood) contributes to the release of PMPs cannot be excluded.

In the present study this shed blood was processed by a cell saver before being returned to the systemic circulation after the bypass procedure had been terminated. This treatment excludes the possibility that systemic blood activation during the bypass procedure is caused by reinfusion of the processed shed blood. On the other hand, we cannot exclude that this reinfusion contributes to blood activation after bypass.

Taken together, although the release of microparticles in vitro from platelets is initiated by complement activation, we found no evidence for a relationship between complement activation and the concentrations of PMPs in systemic blood from patients undergoing CPB. We conclude that complement activation in vivo does not importantly affect the generation of PMPs.

We thank all our colleagues who supported this study and in particular M. C. L. Schaap, R. Baak-Pablo, C. Hau, and H. Everts for excellent technical assistance.

\section{References}

1. Sims PJ, Faioni EM, Wiedmer T, Shattil SJ. Complement proteins C5b-9 cause release of membrane vesicles from the platelet surface that are enriched in the membrane receptor for coagulation factor $\mathrm{Va}$ and express prothrombinase activity. J Biol Chem. 1988;263:1820512.

2. Sims PJ, Wiedmer T, Esmon CT, Weiss HJ, Shattil SJ. Assembly of the platelet prothrombinase complex is linked to vesiculation of the platelet plasma membrane. Studies in Scott syndrome: an isolated defect in platelet procoagulant activity. J Biol Chem. 1989;264:1704957.

3. Tans G, Rosing J, Thomassen MC, Heeb MJ, Zwaal RF, Griffin JH. Comparison of anticoagulant and procoagulant activities of stimulated platelets and platelet-derived microparticles. Blood. 1991;77:2641-8.

4. Wiedmer T, Sims PJ. Participation of protein kinases in complement C5b-9-induced shedding of platelet plasma membrane vesicles. Blood. 1991;78:2880-6.

5. Gilbert GE, Sims PJ, Wiedmer T, Furie B, Furie BC, Shattil SJ. Platelet-derived microparticles express high affinity receptors for factor VIII. J Biol Chem. 1991;266:17261-8.

6. Hoffman M, Monroe DM, Roberts HR. Coagulation factor IXa binding to activated platelets and platelet-derived microparticles: a flow cytometric study. Thromb Haemost. 1992;68:74-8.

7. Holme PA, Brosstad F, Solum NO. Platelet-derived microvesicles and activated platelets express factor Xa activity. Blood Coagul Fibrinolysis. 1995;6:302-10.

8. Nomura S, Shouzu A, Omoto S, Hayakawa T, Kagawa H, Nishikawa M, et al. Effect of cilostazol on soluble adhesion molecules and platelet-derived microparticles in patients with diabetes. Thromb Haemost. 1998;80:388-92.

9. Nomura S, Suzuki M, Katsura K, Xie GL, Miyazaki Y, Miyake T, et al. Platelet-derived microparticles may influence the development of atherosclerosis in diabetes mellitus. Atherosclerosis. 1995;116:235-40.

10. Abrams CS, Ellison N, Budzynski AZ, Shattil SJ. Direct detection of activated platelets and platelet-derived microparticles in humans. Blood. 1990;75:128-38.

11. George JN, Pickett EB, Saucerman S, McEver RP, Kunicki TJ, Kieffer $\mathrm{N}$, et al. Platelet surface glycoproteins. Studies on resting and activated platelets and platelet membrane microparticles in normal subjects, and observations in patients during adult respiratory distress syndrome and cardiac surgery. J Clin Invest. 1986;78:340-8.

12. Katopodis JN, Kolodny L, Jy W, Horstman LL, De Marchena EJ, Tao $\mathrm{JG}$, et al. Platelet microparticles and calcium homeostasis in acute coronary ischemias. Am J Hematol. 1997;54:95-101.

13. Warkentin TE, Hayward CP, Boshkov LK, Santos AV, Sheppard JA, Bode AP, et al. Sera from patients with heparin-induced thrombocytopenia generate platelet-derived microparticles with procoagulant activity: an explanation for the thrombotic complications of heparininduced thrombocytopenia. Blood. 1994;84:3691-9.

14. Lee YJ, Jy W, Horstman LL, Janania J, Reyes Y, Kelley RE, et al. Elevated platelet microparticles in transient ischemic attacks, lacunar infarcts, and multiinfarct dementias. Thromb Res. 1993;72:295-304. 
15. Nomura S, Shouzu A, Nishikawa M, Kokawa T, Yasunaga K. Significance of platelet-derived microparticles in uremia. Nephron. 1993;63: 485.

16. Jy W, Horstman LL, Arce M, Ahn YS. Clinical significance of platelet microparticles in autoimmune thrombocytopenias. J Lab Clin Med. 1992;119:334-45.

17. Holme PA, Solum NO, Brosstad F. Clinical significance of plateletderived microvessels: demonstration of their presence in patients suffering from disseminated intravascular coagulation (DIC). Thromb Haemost. 1993;69:6.

18. Wun T, Paglieroni T, Holland P. Prolonged circulation of activated platelets following plasmapheresis. J Clin Apheresis. 1994;9:10-6.

19. Nieuwland R, Berckmans RJ, McGregor S, Boing AN, Romijn FP, Westendorp RG, et al. Cellular origin and procoagulant properties of microparticles in meningococcal sepsis. Blood. 2000;95:930-5.

20. Nieuwland R, Berckmans RJ, Rotteveel-Eijkman RC, Maquelin KN, Roozendaal KJ, Jansen PG, et al. Cell-derived microparticles generated in patients during cardiopulmonary bypass are highly procoagulant. Circulation. 1997;96:3534-41.

21. Joop K, Berckmans RJ, Nieuwland R, Berkhout J, Romijn FP, Hack $\mathrm{CE}$, et al. Microparticles from patients with multiple organ dysfunction syndrome and sepsis support coagulation through multiple mechanisms. Thromb Haemost. 2001;85:810-20.

22. Berckmans RJ, Nieuwland R, Boing AN, Romijn FP, Hack CE, Sturk A. Cell-derived microparticles circulate in healthy humans and support low grade thrombin generation. Thromb Haemost. 2001;85:639-46.

23. Barry OP, FitzGerald GA. Mechanisms of cellular activation by platelet microparticles. Thromb Haemost. 1999;82:794-800.
24. Hamilton KK, Hattori R, Esmon CT, Sims PJ. Complement proteins C5b-9 induce vesiculation of the endothelial plasma membrane and expose catalytic surface for assembly of the prothrombinase enzyme complex. J Biol Chem. 1990;265:3809-14.

25. Horstman LL, Ahn YS. Platelet microparticles: a wide-angle perspective. Crit Rev Oncol Hematol. 1999;30:111-42.

26. Gemmell CH, Ramirez SM, Yeo EL, Sefton MV. Platelet activation in whole blood by artificial surfaces: identification of platelet-derived microparticles and activated platelet binding to leukocytes as materialinduced activation events. J Lab Clin Med. 1995;125:276-87.

27. Maquelin KN, Berckmans RJ, Nieuwland R, Schaap MC, ten Have K, Eijsman L, et al. Disappearance of glycoprotein $\mathrm{Ib}$ from the platelet surface in pericardial blood during cardiopulmonary bypass. $J$ Thorac Cardiovasc Surg. 1998;115:1160-5.

28. Aldea GS, Soltow LO, Chandler WL, Triggs CM, Vocelka CR, Crockett GI, et al. Limitation of thrombin generation, platelet activation, and inflammation by elimination of cardiotomy suction in patients undergoing coronary artery bypass grafting treated with heparin-bonded circuits. J Thorac Cardiovasc Surg. 2002;123:74255.

29. De Somer F, Van Belleghem Y, Caes F, Francois K, Van Overbeke H, Arnout $\mathrm{J}$, et al. Tissue factor as the main activator of the coagulation system during cardiopulmonary bypass. J Thorac Cardiovasc Surg. 2002;123:951-8.

30. Kestin AS, Valeri CR, Khuri SF, Loscalzo J, Ellis PA, MacGregor H, et al. The platelet function defect of cardiopulmonary bypass. Blood. 1993;82:107-17. 